\title{
Time-Dependent Density Functional Theory of Classical Fluids
}

\author{
Garnet Kin-Lic Chan \\ Department of Chemistry and Chemical Biology, Cornell University, Ithaca, New York 14853-1301, USA \\ Reimar Finken \\ II. Institut für Theoretische Physik, Universität Stuttgart, Pfaffenwaldring 57/III, 70550 Stuttgart, Germany
}

(Received 9 February 2004; published 11 May 2005)

\begin{abstract}
We establish a rigorous time-dependent density functional theory of classical fluids for a wide class of microscopic dynamics. We obtain a stationary action principle for the density. We further introduce an exact practical scheme, to obtain hydrodynamical effects in density evolution, that is analogous to the Kohn-Sham theory of quantum systems. Finally, we show how the current theory recovers existing phenomenological theories in an adiabatic limit.
\end{abstract}

DOI: 10.1103/PhysRevLett.94.183001

PACS numbers: 31.15.Ew, 64.10.+h

In the usual classical density functional theory, properties of an equilibrium system, such as the free energy, are expressed as functionals of the equilibrium one-particle density $\rho_{0}(\mathbf{r})$. The existence of the functionals is guaranteed by the Hohenberg-Kohn-Mermin theorem [1,2] which, for a fixed interparticle interaction $w$, establishes an invertible map between $\rho_{0}$ and the external potential $v$, $\rho_{0}(\mathbf{r}) \leftrightarrow v(\mathbf{r})$, thus determining the Hamiltonian and all equilibrium properties of the system from $\rho_{0}$. Density functional theory is now widely used as a quantitative theory of equilibrium states under diverse conditions $[3,4]$.

Recently there have been attempts, along phenomenological lines, to extend equilibrium density functional theory to nonequilibrium systems [5-8]. (By nonequilibrium, we include also out-of-equilibrium systems in the presence of time-dependent fields.) To establish a density functional theory of dynamical phenomena, we require an invertible map between the time-dependent density $\rho(\mathbf{r}, t)$ and the distribution function $f(\mathbf{x}, t)$ ( $\mathbf{x}$ denotes the coordinates of all $N$ particles, $\left.\mathbf{r}_{1}, \ldots, \mathbf{r}_{N}, \mathbf{p}_{1}, \ldots \mathbf{p}_{N}\right)$, thus allowing all time-dependent properties of the system to be expressed as functionals of $\rho(\mathbf{r}, t)$. In quantum mechanical systems, Runge and Gross showed how the corresponding map between $\rho(\mathbf{r}, t)$ and the time-dependent wave function $\Psi\left(\mathbf{r}_{1}, \ldots, \mathbf{r}_{N}, t\right)$ could be constructed with the external potential as an intermediary, $\rho(\mathbf{r}, t) \leftrightarrow v(\mathbf{r}, t) \leftrightarrow$ $\Psi\left(\mathbf{r}_{1}, \ldots, \mathbf{r}_{N}, t\right)$ [9]. In this Letter, we use the ideas of Runge and Gross to develop a rigorous time-dependent density functional theory of classical systems. The structure of our discussion is as follows. First, we construct the classical analogue of the Runge-Gross theorem, establishing the map $\rho(\mathbf{r}, t) \leftrightarrow v(\mathbf{r}, t) \leftrightarrow f(\mathbf{x}, t)$, both for Hamiltonian and non-Hamiltonian systems. Next, restricting our discussion to Hamiltonian systems, we propose an action formalism that yields the exact time-dependent density via a stationary principle. Finally, we describe a practical approximation for modeling hydrodynamic phenomena in the theory, through an auxiliary construction that resembles the Kohn-Sham theory of quantum systems [10].

To establish the invertible map $\rho(\mathbf{r}, t) \leftrightarrow v(\mathbf{r}, t) \leftrightarrow$ $f(\mathbf{x}, t)$, let us first consider a system with underlying Hamiltonian microscopic dynamics. For an $N$ particle system with a time-dependent external potential $v$, and a general interparticle interaction $w$, the Hamiltonian takes the form

$$
H=\sum_{i} \frac{\mathbf{p}_{i}^{2}}{2}+\sum_{i} v\left(\mathbf{r}_{i}, t\right)+\sum_{i>j>\ldots} w\left(\mathbf{r}_{i}, \mathbf{r}_{j}, \ldots\right),
$$

where unit mass has been chosen for simplicity. The microscopic equations of motion are $\dot{\mathbf{r}}_{i}=\partial H / \partial \mathbf{p}_{i} ; \dot{\mathbf{p}}_{i}=$ $-\partial H / \partial \mathbf{r}_{i}$, and the equation of motion for $f$ is the Liouville equation $\partial f / \partial t=\hat{L} f$, where the Liouville operator is $\hat{L}=-\dot{\mathbf{x}} \cdot \nabla_{\mathbf{x}}$.

For a fixed initial distribution function $f\left(\mathbf{x}, t_{0}\right)$, we can solve the Liouville equation with various external potentials $v$, establishing the map $v \rightarrow f$, up to a purely timedependent constant in $v$. Since the Liouville equation is deterministic, this map may be inverted (up to a purely time-dependent constant) to yield $v \leftrightarrow f$; informally, this is because different potentials $v$ immediately produce different time derivatives of $f$. Now for each $f$ obtained in this way, we form the one-particle density as the phase space average $\rho(\mathbf{r}, t)=\int \hat{\rho} f d \mathbf{x}$, thereby establishing the map $\rho \leftarrow v \leftrightarrow f$. To complete our theory, we need to establish the inverse map $\rho \rightarrow v$, i.e., to show that different densities necessarily result from different external potentials.

First, we demonstrate that $v \leftrightarrow \mathbf{j}$, where $\mathbf{j}$ is the oneparticle current density. Defining $\mathbf{j}$ as the phase space average $\mathbf{j}(\mathbf{r}, t)=\int \hat{\mathbf{j}} f d \mathbf{x}$, where $\hat{\mathbf{j}}=\Sigma_{i} \mathbf{p}_{i} \delta\left(\mathbf{r}_{i}-\mathbf{r}\right)$, the map $v \rightarrow \mathbf{j}$ can be constructed in the same way as for the density above. To establish $\mathbf{j} \rightarrow v$, we use the equation of motion for $\mathbf{j}$, which is obtained from the Liouville equation as 


$$
\begin{aligned}
& \frac{\partial \mathbf{j}}{\partial t}=-\nabla_{\mathbf{r}} \cdot \int \sum_{i} \mathbf{p}_{i} \mathbf{p}_{i} \delta\left(\mathbf{r}_{i}-\mathbf{r}\right) f d \mathbf{x}-\rho \nabla_{\mathbf{r}} v_{\text {eff }} \\
& \equiv-\nabla_{\mathbf{r}} \cdot \int \hat{\sigma} f d \mathbf{x}-\rho \nabla_{\mathbf{r}} v_{\text {eff }}, \\
& v_{\text {eff }}=v+\int \sum_{i>j>\ldots} w\left(\mathbf{r}_{i}, \mathbf{r}_{j}, \ldots\right) \hat{\rho} f d \mathbf{x} .
\end{aligned}
$$

We now show that the current densities $\mathbf{j}$, $\mathbf{j}^{\prime}$ obtained by evolving from a fixed initial distribution function $f\left(t_{0}\right)$, under the influence of two different external potentials $v$ and $v^{\prime}$ are necessarily different over any finite time interval. Let us assume that $v$ and $v^{\prime}$ are identical up to some time $t_{0}$. At time $t_{0}$, let the external potentials begin to differ in their $n$th derivative,

$$
\frac{\partial^{n}}{\partial t^{n}}\left(v-v^{\prime}\right)=u(\mathbf{r}, t)
$$

where $u(\mathbf{r}, t)$ is not a purely time-dependent constant. Repeated differentiation of Eq. (2) yields

$$
\left(\frac{\partial}{\partial t}\right)^{n+1}\left(\mathbf{j}-\mathbf{j}^{\prime}\right)=-\rho \nabla_{\mathbf{r}} u
$$

which, as $u$ is not a purely time-dependent constant, implies that a change in $v$ of the form (4) necessarily leads to a change in the current density $\mathbf{j}$ infinitesimally after time $t_{0}$, establishing the map from $\mathbf{j} \rightarrow \boldsymbol{v}$.

Now to obtain a similar map $\rho \rightarrow v$, we show that systems which have different current densities must have different densities. From the continuity equation

$$
\frac{\partial \rho}{\partial t}=-\nabla_{\mathbf{r}} \cdot \mathbf{j}
$$

and Eq. (5), we find

$$
\left(\frac{\partial}{\partial t}\right)^{n+2}\left(\rho-\rho^{\prime}\right)=\nabla_{\mathbf{r}} \cdot\left(\rho \nabla_{\mathbf{r}} u\right) .
$$

Now consider the integral $\int u \nabla_{\mathbf{r}} \cdot\left(\rho \nabla_{\mathbf{r}} u\right) d \mathbf{r}$. By the divergence theorem, $\int u \nabla_{\mathbf{r}} \cdot\left(\rho \nabla_{\mathbf{r}} u\right) d \mathbf{r}=-\int \rho\left(\nabla_{\mathbf{r}} u\right)^{2} d \mathbf{r} \neq 0$, establishing that the right-hand side of Eq. (7) is nonvanishing [11]. Thus, a change in $v$ of the form (4) necessarily leads to a change in $\rho$ infinitesimally after time $t_{0}$, which establishes the map from $\rho \rightarrow v$. Together with $\rho \leftarrow v \leftrightarrow$ $f$ established earlier, this completes the map $\rho \leftrightarrow v \leftrightarrow f$. Thus for a given initial distribution function, the subsequent time-dependent density uniquely determines the subsequent external potential (up to a time-dependent constant), and $N$ particle distribution function, and therefore all the time-dependent properties of the system may be expressed as functionals of the time-dependent density $\rho$ [13].

So far, this establishes a time-dependent density functional theory for systems with underlying Hamiltonian dynamics. In many models of fluids, it is common to assume that the particles are governed by non-
Hamiltonian dynamics, where the general equation of motion takes the form $\dot{\mathbf{x}}=\mathbf{v}(\mathbf{x})$. So long as $\mathbf{v}(\mathbf{x})$ depends linearly on $v$ (e.g., contains a term such as $\nabla_{\mathrm{r}} v$ or $c v$ ) the general structure of the Runge-Gross proof holds, and a time-dependent density functional theory may be constructed. As an example, we consider explicitly the case of Brownian dynamics. Here, the equations of motion are given by $\dot{\mathbf{r}}_{i}=\mathbf{p}_{i} ; \dot{\mathbf{p}}_{i}=-\nabla_{\mathbf{r}_{i}} v-\gamma \mathbf{p}_{i}+\eta_{i}$, where $\gamma$ is a friction coefficient and $\eta_{i}=\left(\eta_{i}^{x}, \eta_{i}^{y}, \eta_{i}^{z}\right)$ is a Gaussian noise vector satisfying $\left\langle\eta_{i}\right\rangle=0$ and $\left\langle\eta_{i}^{\alpha}(t) \eta_{j}^{\beta}\left(t^{\prime}\right)\right\rangle=$ $2 D \delta_{i j} \delta_{\alpha \beta} \delta\left(t-t^{\prime}\right)$. We obtain Liouville's equation by explicit differentiation of $f(\mathbf{x})$ (see, e.g., Refs. [14,15]): this yields

$$
\frac{\partial f}{\partial t}=-\nabla_{\mathbf{x}} \cdot(\dot{\mathbf{x}} f)+D \nabla_{\mathbf{p}}^{2} f
$$

where the additional term $D \nabla_{\mathbf{p}}^{2} f$ arises from the contribution of the random variable to the Ito stochastic derivative [16]. Taking averages yields two equations, the continuity Eq. (6) and a current equation

$$
\frac{\partial \mathbf{j}}{\partial t}=-\nabla_{\mathbf{r}} \cdot \int \hat{\sigma} f d \mathbf{x}-\rho\left(\nabla_{\mathbf{r}} v_{\text {eff }}+\zeta\right)+\gamma \mathbf{j},
$$

where following Dean [6] we have introduced a noise field $\zeta(\mathbf{r}, t)=\rho^{-1 / 2}(\mathbf{r}, t) \eta(\mathbf{r}, t), \quad\left\langle\eta_{i}^{\alpha}(\mathbf{r}, t) \eta_{j}^{\beta}\left(\mathbf{r}^{\prime}, t^{\prime}\right)\right\rangle=$ $2 D \delta_{i j} \delta_{\alpha \beta} \delta\left(t-t^{\prime}\right) \delta\left(\mathbf{r}-\mathbf{r}^{\prime}\right)$, which averages noise contributions from different particles in the mean motion of the density. As expected, in addition to normal Newtonian terms, the Euler equation (9) also contains friction and random noise elements. Repeated differentiation of $\left(\rho-\rho^{\prime}\right),\left(\mathbf{j}-\mathbf{j}^{\prime}\right)$ yields (5) and (7) immediately and the map $\rho \leftrightarrow v \leftrightarrow f$, thus establishing the Brownian density functional theory.

Thus, for fairly general forms of the microscopic equations of motion, a time-dependent density functional theory is easily established. The corresponding time-evolved density is obtained through the solution of two hydrodynamic equations, the continuity equation $\partial \rho / \partial t=-\nabla_{\mathbf{r}} \cdot \mathbf{j}$ and a current equation of the general form

$$
\frac{\partial \mathbf{j}}{\partial t}=\mathbf{P}[\rho]
$$

of which Eqs. (2) and (9) are particular examples. From the point of view of practical calculation, the relevant functional to approximate in this theory is $\mathbf{P}[\rho]$.

Before proceeding in that direction, however, we also would like to have a variational formulation of the theory, i.e., a stationary action principle analogous to the variational principle in equilibrium density functional theory. Such a principle would be expected to exist for systems with underlying Hamiltonian dynamics. We now formulate classical time-dependent density functional theory in terms of a stationary action principle, restricting ourselves henceforth to systems with Hamiltonian dynamics. 
We first note that the interplay between action principles in density functional theory and causality can be quite subtle $[17,18]$. In our treatment, we neglect these difficulties for now and employ a simple formalism which leads to symmetric response functions. From the Lagrangian density $\mathcal{L}(\mathbf{x}, \dot{\mathbf{x}})=T-V=\Sigma_{i} \dot{\mathbf{r}}_{i}^{2} / 2-$ $\Sigma_{i} v\left(\mathbf{r}_{i}\right)-\Sigma_{i>j>\ldots} w\left(\mathbf{r}_{i}, \mathbf{r}_{j}, \ldots\right)$, we can form an action for the distribution function, $S[f, v]$,

$$
\begin{gathered}
S[f, v]=\int_{t_{0}}^{t_{f}} \int L f d \mathbf{x} d t \\
=\int_{t_{0}}^{t_{f}}\left[\int T f d \mathbf{x}-\int \rho\left(v+w_{\mathrm{xc}}\right) d \mathbf{r}\right] d t, \\
w_{\mathrm{xc}}(\mathbf{r}, t) \equiv \int \sum_{i>j>\ldots} w\left(\mathbf{r}_{i}, \mathbf{r}_{j}, \ldots\right) \hat{\rho} f d \mathbf{x} .
\end{gathered}
$$

$S[f, v]$ is stationary with respect to variations in the trajectories of the particles around the classical stationary action trajectories in the potential $v$; consequently, it is stationary with respect to norm-conserving variations (induced by microscopic variations in the trajectories) in $f$ around the solution $f_{v}=f_{v}(\mathbf{x}, t)$ of the Liouville equation in the potential $v$. This classical action principle is an analogue of the Dirac-Frenkel quantum action [9], although, unlike in the quantum action, $S[f, v]$ does not itself vanish at the stationary points.

We need to convert the action functional of the distribution function $S[f, v]$ into an action functional of the density. This can be done through a constrained search $[19,20]$

$$
S[\rho, v]=\operatorname{stat}_{\hat{\rho} f d \mathbf{x}=\rho} S[f, v]=A[\rho]-\int \rho v d \mathbf{r},
$$

where above "stat" indicates that $S[f, v]$ is made stationary with respect to variations in $f$, under the constraint that $\int \hat{\rho} f d \mathbf{x}=\rho$. If the density in Eq. (14) is that obtained from $f_{v}\left(\mathbf{x}, t_{0}\right)$, i.e., $\rho=\rho_{v}=\int \hat{\rho} f_{v} d \mathbf{x}$, then the stationary $f$ in the constrained search is $f=f_{v}\left(\mathbf{x}, t_{0}\right)$. Assuming differentiability of $S[\rho, v]$, then $S[\rho, v]$ is stationary at $\rho_{v}$, and $A[\rho]$ satisfies the Euler-Lagrange equation

$$
\left(\frac{\delta A[\rho]}{\delta \rho}\right)_{\rho_{v}}=v
$$

This establishes a stationary principle for time-dependent density functional theory.

We have now constructed two equivalent formalisms for the time-dependent density functional theory. We return then to the problem of finding practical approximate functionals to model hydrodynamic and correlation effects. In the usual equilibrium density functional theory, we divide the free energy functional $F[\rho]$ into the contribution of a noninteracting, "ideal" component $F_{\text {id }}[\rho]$, for which the exact expression is known, and an excess functional $F_{\text {xc }}[\rho]$ which must be approximated. A similar strategy may be adopted in the time-dependent case. Restricting ourselves to Hamiltonian dynamics, the Euler equation functional $\mathbf{P}[\rho]$ in the equation of motion Eq. (10) may be decomposed into parts, along the lines of Eq. (2),

$$
\begin{gathered}
\mathbf{P}(\mathbf{r}, t)[\rho]=-\nabla_{\mathbf{r}} \cdot \sigma[\rho]-\rho\left(\nabla_{\mathbf{r}} v+\nabla_{\mathbf{r}} w_{\mathrm{xc}}[\rho]\right), \\
\sigma(\mathbf{r}, t)[\rho]=\int \hat{\sigma} f[\rho] d \mathbf{x},
\end{gathered}
$$

where $f[\rho]$ is defined through the map $\rho \rightarrow f$. The two unknown components are the stress tensor $\sigma[\rho]$, which models hydrodynamic effects, and $w_{\mathrm{xc}}[\rho]$, which takes into account particle correlations. We focus first on $\sigma[\rho]$. As argued, we can approximate $\sigma[\rho]$ by first separating out a noninteracting (ideal gas) contribution: thus we have $\sigma[\rho]=\sigma_{\mathrm{ni}}[\rho]+\sigma_{\mathrm{xc}}[\rho]$, where $\sigma_{\mathrm{ni}}[\rho]$ is the stress functional of a corresponding noninteracting reference system constrained to have the interacting density $\rho$ and $\sigma_{\mathrm{xc}}[\rho]$ denotes the excess contributions due to interactions.

Although $\sigma_{\mathrm{ni}}[\rho]$ is the stress tensor for a noninteracting system, we do not know its functional form explicitly. A similar situation arises already in the quantum theory of equilibrium systems, where we do not know the explicit form of the kinetic energy functional in noninteracting, inhomogeneous, systems. However, we can adopt a similar solution as in the quantum case. We note that $\sigma_{\text {ni }}[\rho]$ can be calculated trivially if we know the noninteracting one-particle reduced distribution function $f_{\mathrm{ni}}^{1}(\mathbf{r}, \mathbf{p}, t)=$ $\int \Sigma_{i} \delta\left(\mathbf{p}_{i}-\mathbf{p}\right) \hat{\rho} f_{\mathrm{ni}} d \mathbf{x}$, since

$$
\sigma_{\mathrm{ni}}(\mathbf{r}, t)[\rho]=\int \mathbf{p} \mathbf{p} f_{\mathrm{ni}}^{1}(\mathbf{r}, \mathbf{p}, t) d \mathbf{p} .
$$

Consequently, we introduce an auxiliary, noninteracting, "Kohn-Sham" reference system, which evolves in the presence of an effective potential $v_{\mathrm{ni}}(\mathbf{r}, t)[\rho]$ so as to always reproduce the density of the interacting system. From the $f^{1}(\mathbf{r}, \mathbf{p}, t)$ of this reference, we may easily evaluate $\sigma_{\text {ni }}[\rho]$ at the interacting density $\rho$.

We now need to determine the effective potential of the noninteracting system $v_{\text {ni }}(\mathbf{r}, t)[\rho]$ that constrains its density to be identical to that of the interacting system. This can be done within the action formalism we have developed. Separating the action $A[\rho]$ as

$$
A[\rho]=T[\rho]-W_{\mathrm{xc}}[\rho]-\int \rho v d \mathbf{r},
$$

where $T[\rho]=\iint T f[\rho] d \mathbf{x} d t, W_{\text {хс }}[\rho]=\iint \rho w_{\text {xс }}[\rho] d \mathbf{r} d t$, the Euler-Lagrange Eq. (15) for the interacting system is

$$
\left(\frac{\delta T}{\delta \rho}\right)_{\rho_{v}}-\left(\frac{\delta W_{\mathrm{xc}}}{\delta \rho}\right)_{\rho_{v}}-v=0,
$$

while that of the noninteracting system is

$$
\left(\frac{\delta T_{\mathrm{ni}}}{\delta \rho}\right)_{\rho_{v_{\mathrm{ni}}}}-v_{\mathrm{ni}}=0 .
$$


Note that $T_{\text {ni }}[\rho] \neq T[\rho]$, since $T_{\text {ni }}[\rho]$ is the kinetic energy of the noninteracting system with density $\rho$. It follows that for the noninteracting system to yield the interacting density $\rho[v]$ as desired, the effective potential $v_{\mathrm{ni}}(\mathbf{r}, t)$ must satisfy

$$
\begin{gathered}
v_{\mathrm{ni}}(\mathbf{r}, t)[\rho]=v_{\mathrm{xc}}(\mathbf{r})[\rho]+v, \\
v_{\mathrm{xc}}(\mathbf{r}, t)[\rho]=\left(\frac{\delta A_{\mathrm{xc}}}{\delta \rho}\right)_{\rho[v]},
\end{gathered}
$$

where we have introduced an excess action functional $A_{\mathrm{xc}}[\rho]=T[\rho]-T_{\mathrm{ni}}[\rho]-W_{\mathrm{xc}}[\rho]$ that contains a pure correlation component $\left(W_{\mathrm{xc}}[\rho]\right)$, and the difference between the real and noninteracting hydrodynamics $(T[\rho]-$ $T_{\text {ni }}[\rho]$ ). Comparing with the hydrodynamic equation (16), we obtain the connection $v_{\mathrm{xc}}=\nabla_{\mathbf{r}} \cdot \sigma_{\mathrm{xc}}+w_{\mathrm{xc}}$.

To summarize, to model the hydrodynamic behavior of our system due to the functional $\sigma[\rho]$, we introduced a noninteracting "Kohn-Sham" reference system in the presence of an effective potential $v_{\mathrm{ni}}(\mathbf{r}, t)[\rho]$ that reproduces the exact interacting density at all times. Then to time evolve the interacting density, we need only time evolve the noninteracting system (e.g., by a simple noninteracting molecular dynamics simulation, or through the equation of motion of $f_{\mathrm{ni}}^{1}$ ) in the presence of the effective potential $v_{\mathrm{ni}}$, and the resulting time-evolved noninteracting density is identical to the full interacting density. All this is possible in terms of a single, approximable functional, the excess action $A_{\text {xc }}[\rho]$, whose functional derivative $v_{\mathrm{xc}}[\rho]$ yields the unknown part of the effective potential $v_{\mathrm{ni}}$.

It remains to characterize the excess action $A_{x c}[\rho]$ in more detail. Some insight can be gained from an integration where we scale the particle interaction $w$. Consider the functional $A^{\lambda}[\rho, v]$, where $\lambda$ is a parameter that multiplies the interaction $w$. Then, $A[\rho, v]=A^{1}[\rho, v], A_{\text {ni }}[\rho, v]=$ $A^{0}[\rho, v]$, and since $A[\rho, v]-A_{\mathrm{ni}}[\rho, v]=A_{\mathrm{xc}}[\rho]$ we have

$$
A_{\mathrm{xc}}[\rho]=\int_{0}^{1} \frac{\partial A^{\lambda}}{\partial \lambda} d \lambda=\iiint_{0}^{1} w \hat{\rho} f^{\lambda} d \lambda d \mathbf{x} d t,
$$

where $f^{\lambda}(\mathbf{x}, t)[\rho]$ is defined by $\rho \rightarrow f$ with interaction $\lambda w$, and we have used the Hellmann-Feynman theorem in Eq. (24). Now, the same expression (24), without time integration, holds in equilibrium density functional theory, but with $A_{\mathrm{xc}}[\rho]$ replaced by the excess free energy $F_{\text {ex }}[\rho]$ and $f=f_{0}$. Thus we obtain the clear interpretation that the excess action $A_{\mathrm{xc}}[\rho]$ is simply the time-averaged excess free energy functional.

A simple approximation for $A_{\mathrm{xc}}[\rho]$ is to use the representation of the excess functional $F_{\text {ex }}[\rho]$ for equilibrium density functional theory. Such an "adiabatic" approximation has no time correlation, and $v_{\mathrm{ni}}[\rho]$ is a local function of time. It is this approximation that underlies recent phenomenological dynamic density functional theories. For example, the adiabatic approximation for the Euler equation in the case of overdamped dynamics reduces to the familiar form $[6,7,21]$

$$
\frac{\partial \rho}{\partial t}=\nabla_{\mathbf{r}}\left[\rho \cdot \nabla_{\mathbf{r}}\left(v+\frac{\delta F_{\mathrm{ex}}}{\delta \rho}\right)+\zeta \rho\right]+D \nabla_{\mathbf{r}}^{2} \rho .
$$

To go beyond the adiabatic approximation, we need to introduce memory effects. One way to do so is through the memory function formalism employed in modecoupling theory, and some initial attempts along these lines in the quantum theory are described, for example, in Ref. [22].

In conclusion, we have constructed a rigorous timedependent density functional theory for classical systems, for a wide class of microscopic dynamics. Within this density functional theory, the time-evolved density may be obtained in one of three ways: through the hydrodynamic equations (2) and (6), through a stationary action principle (15), or through evolving a noninteracting reference system in the presence of an effective potential. In situations where hydrodynamic effects are important, the last scheme is the most practical approach. The effective potential is then the functional derivative of an excess action functional, and the excess action is the timedependent generalization of the excess free energy, to which it reduces in an adiabatic limit.

[1] P. Hohenberg and W. Kohn, Phys. Rev. 136, B864 (1964).

[2] N. D. Mermin, Phys. Rev. 137, A1441 (1965).

[3] H. Löwen, J. Phys. Condens. Matter 15, V1 (2003).

[4] R. Evans, Fundamentals of Inhomogeneous Fluids (Dekker, New York, 1992).

[5] W. Dieterich, H. L. Frisch, and A. Majhofer, Z. Phys. B 78, 317 (1990).

[6] D. S. Dean, J. Phys. A 29, L613 (1996).

[7] U.M. B. Marconi and P. Tarazona, J. Chem. Phys. 110, 8032 (1999).

[8] J. Dzubiella and C. N. Likos, J. Phys. Condens. Matter 15, L147 (2003).

[9] E. Runge and E. K. U. Gross, Phys. Rev. Lett. 52, 997 (1984).

[10] W. Kohn and L. J. Sham, Phys. Rev. 140, A1133 (1965).

[11] More precise mathematical conditions are established in [12].

[12] A. K. Dhara and S. K. Ghosh, Phys. Rev. A 35, 442 (1987).

[13] We restrict our densities to the $v$ representable set.

[14] J. D. Ramshaw, Europhys. Lett. 59, 319 (2002).

[15] G. S. Ezra, J. Math. Chem. 32, 339 (2002).

[16] C.W. Gardiner, Handbook of Stochastic Methods (Springer, New York, 1985).

[17] R. van Leuuwen, Phys. Rev. Lett. 80, 1280 (1998).

[18] R. van Leuuwen, Int. J. Mod. Phys. B 15, 1969 (2001).

[19] M. Levy, Proc. Natl. Acad. Sci. U.S.A. 76, 6062 (1979).

[20] A. Görling, Phys. Rev. A 59, 3359 (1999).

[21] The noise term can be eliminated through redefinition of $F_{\text {ex }}$ to obtain the formulation in [7].

[22] C. A. Ullrich and G. Vignale, Phys. Rev. B 65, 245102 (2002). 